\title{
Implementation of massive transfusion protocol (MTP) for trauma
}

\author{
R.N. Ellawala ${ }^{1,2}$, K. Gonsalkorala ${ }^{2}$ I.S. Wijesiriwardena ${ }^{2}$ \\ 1 Department of Surgery, Faculty of Medicine, General Sir John Kotelawala Defence University, Ratmalana, \\ Sri Lanka \\ 2 Colombo South Teaching Hospital, Sri Lanka
}

\begin{abstract}
\section{Introduction}

Massive transfusion, the administration of 10 or more units of packed red blood cells (RBC) in less than 24 hours, can be a lifesaving therapy in the treatment of severe bleeding following injury. The rapid delivery and administration of large numbers of Packed Red Blood cells (PRBC), along with sufficient plasma and platelets is frequently a disorderly process. Establishment of MTP addresses this issue and has shown to reduce mortality and reduce the effects of coagulopathy associated with trauma.
\end{abstract}

\section{Methods}

Following a preliminary discussion with all the stake holders including the administrators, we implemented a protocol which supports the ratio of 1:1:1, of PRBC: FFP(Fresh Frozen Plasma) : Platelet. Each step of the process, from activation of protocol to termination was defined. The contents of boxes 1, 2 and 3 were predetermined.

\section{Conclusion}

We hope to encourage the use of MTP in other centres in the country to achieve better results with post-trauma bleeding patients. The effectivenes of implementing such a protocol depends on the understanding and collaboration of each member responsible.

\section{Introduction}

Massive transfusion protocol (MTP) has been designed to facilitate delivery of large volumes of blood and blood products to a patient who requires them to prevent exsanguination. This is considered as an essential practice around the globe [1]. Although MTP goes hand

Correspondence: Ranjith Nanda Ellawala

E-mail: ranjithellawala@yahoo.com in hand with trauma care, the protocol could be used in other situations where bleeding is catastrophic. Two essential components of the protocol are rapidity and smooth flow of delivery of blood and blood products and the predefined ratio of these products.

Though blood transfusion is not without adverse effects, it may be necessary to replace large volumes to save lives. When the volume of blood transfused during 24 hours equals to one blood volume (5liters), it is considered as a massive transfusion. Alternatively massive transfusion is also defined as $50 \%$ of the volume replaced within 3 hours, or when the rate of replacement is $>150 \mathrm{ml} / \mathrm{min}[1,2]$.

There is evidence to show that when massive transfusion is used with the blood component ratio equal to 1:1:1 of packed red blood cells: fresh frozen plasma: apheresis donor platelets (PRBC: FFP: Platelets) is associated with better survival. Most of the trauma centres around the globe use ratios similar to this $[3,4,5]$.

\section{Haemorrhagic shock}

Hemorrhagic shock is a leading preventable cause of death following trauma. One third of trauma deaths occur due to victims bleeding to death within the first few hours after injury. Bleeding ranks as the 2 nd cause of trauma deaths in a hospital, only second to head injuries [6]. The physiological changes which follow bleeding will be irreversible when it is not controlled promptly. The treatment of bleeding is to stop bleeding as early as possible. In most situations this is achieved through surgical interventions. Although angioembolization is effective in certain situations, it is not readily available in the emergency setting in Sri Lanka as a regular therapeutic option. It is necessary that the replacement of what was lost is done appropriately to maintain the functions of the vital organs. This may mean transfusion of large numbers of blood units within 
a short period.

\section{Trauma Coagulopathy}

A dying patient following an injury will have a combination of three conditions i.e. hypothermia, acidosis and coagulopathy. These conditions are collectively called the "Triad of Death". Presence of any of the above three factors mentioned has the ability to potentiate each other. Once established, these will lead to death. Of all these three factors, coagulopathy is the most important factor contributing to mortality. Its aetiology in trauma has been described as occurring due to dilution, acidosis, consumption and hypothermia. Acute Trauma Coagulopathy, a new entity introduced in 2003, has a different aetiology [7]. Tissue injury and shock following an injury leads to activation of the protein $\mathrm{C}$ pathway which will in turn lead to systemic anticoagulation and hyperfibrinolysis. The coagulopathy of trauma has received much attention recently and is associated with a fourfold rise in mortality.

\section{Damage Control Resuscitation (DCR)}

The DCR concept, which emerged recently was developed to counteract the coagulopathy associated with trauma. It consists of [8];

1. Steps to achieve control of bleeding- this is achieved with surgery

2. Minimal crystalloid infusion-traditional treatment with large volumes of crystalloids and colloids is no longer acceptable in this situation $[9,10]$

3. Early blood and blood product transfusion with predefined ratio. (Early Haemostatic Resuscitation)

4.Permissive Hypotension[11]-maintaining systolic blood pressure low in the presence of no contraindications till the bleeding is arrested.

\section{Justification}

MTP is commonly used in trauma centres in USA, Europe, Australia[1] and New Zealand. Medline searches did not reveal use of MTP or any publications in the South East Asia region except in Singapore and in Thailand. It is the authors' experience that when large volumes of blood and blood products are required within a short period in our hospitals, there could be considerable delays and confusion leading to suboptimal care. Hence we implemented MTP at CSTH.

\section{Massive Transfusion Protocol(MTP)}

MTP is a planned schedule of blood component delivery, is simple to follow and components received are predictable.

Use of MTP has shown not only significant improvement in survival, especially after blunt trauma, but also decrease in early crystalloid usage and improvement in coagulation parameters. Studies have confirmed the survival benefit of MTP. Dramatic reduction in many of the complications including organ failure has been observed in critically injured patients receiving massive transfusions $[3,5,12,13]$.

Rapid processing and preparation of a large amount of blood and blood products in a short period of time requires planning and coordination in the blood bank to ensure delivery of these products in an immediate and sustained manner. There is an efficient network of blood transfusion services in Sri Lanka, but when considering the delivery of large amounts of blood during a short period at an institutional level, it is the experience of the authors that there could be considerable delays.

The reasons for delay are numerous. Delay could occur at each and every step, from the time of request, to delivery the emergency care unit. The delay could be more pronounced if blood components are requested separately. Hence the value of a protocol to administer massive volumes of blood and blood products cannot be overemphasized $[2,8,10]$.

There was no massive transfusion protocol practiced in Sri Lanka in 2013. The authors' objective was to introduce a massive transfusion protocol after studying the protocols which are being practiced in major trauma centres across the globe.

\section{Methodology}

Implementation of the Massive Transfusion Protocol at Colombo South Teaching Hospital (CSTH).

We implemented the MTP at CSTH in 2013. Once we decided to implement the protocol, a meeting was held with all stakeholders, which included the consultant 
surgeon, medical officer in charge and sister in charge, representing the Accident Service, consultant in Transfusion Medicine, medical officer in charge and sister in charge, representing the Blood Bank and the hospital administrators.

Consensus were achieved with regard to;

1. Issue of uncrossmatched blood on medical officer's request

2. Rapid issue of blood products.

3. Logistics in transporting boxes containing blood and blood products.

This meeting was followed by a presentation on the subject to the medical officers and the consultants of CSTH. Ethical clearance was obtained from the CSTH Ethical Committee and the protocol was implemented.

Once the injured patient is brought to the resuscitation room of the Accident Service (AS) of CSTH, primary survey and resuscitation occurs simultaneously. This process is similar to those practiced in most of the large hospitals (teaching, provincial, some base hospitals with Emergency Care Units) in Sri Lanka. This is carried out by medical officers at the accident Service.

Identification of patients who requires MTP is difficult. It is the decision of the surgeon on duty to activate the MTP, based on clinical judgment. To help in decision making, several scoring systems are available and practiced in some recognized centres abroad. One such simple scoring system is the Assessment of Blood Consumption (ABC).

According to this scoring system a score of 2 or more predicts massive transfusion requirement. The score is calculated using 4 indices measured on admission [14]:
1. Penetrated injury
Y 1 N 0
2. Systolic BP of $<90 \mathrm{mmHg}$
Y $1 \mathrm{~N} 0$
3. Pulse $>120 / \mathrm{min}$
Y 1 N0
4. Positive FAST
Y1 N 0

(FAST-Focused Abdominal Sonography for Trauma)

The presence of any of theparameters stated above will score one point each and absence will score 0 . Penetrating injuries are uncommon in our setup.
Although ultrasound is being used widely for trauma in Sri Lanka, it may not be available immediately in the emergency treatment room. Hence the decision is made on systolic blood pressure and pulse rate in most instances. It may takes approximately 15 minutes or even more for complete assessment of the degree of blood loss and its progression.

\section{Steps in activation of the Massive Transfusion Protocol (MTP) at Colombo South Teaching Hospital}

Initial fluid replacement done with crystalloid (normal saline or Hartman's solution) followed by two units or more of uncross-matched blood in the presence of the following situations

1. Unrecordable blood pressure with obvious major multiple injuries

2. Unrecordable blood pressure with no obvious multiple injuries which does not respond to fluid

3. If initial systolic BP 70 or less and no dramatic response to crystalloid infusion .

4. If obvious massive bleeding is evident.

\section{Protocol activation and delivery of blood products}

- Once the uncrossmatched blood transfusion is initiated, continued assessment will indicate the necessity of activating the Massive Transfusion Protocol (MTP). With available clinical findings combined with the score of $\mathrm{ABC}$ scoring system in difficult situations, MTP is activated.

- The activation is done by a medical officer, surgical registrar or consultant surgeon by phoning the blood bank informing the medical officer of the blood bank.

- It is possible that transfusion of cross-matched blood may be done prior to MTP activation.

The accompanying flow chart indicates how the delivery of boxes containing blood and blood products, takes place ( Figure 1.).

Recommended follow up laboratory assessment is done every 6 hours for the first 12 hours post stabilisation and then at 24 hours post stabilisation. The majority of 
institutions in Sri Lanka with limited laboratory facilities are not able to perform laboratory tests in an ideal manner. Hence the protocol should be adjusted accordingly. from achieving haemostasis, replacement of large amounts of blood, requires a plan. The protocol involving the use of fixed proportion of blood components avoids delay, confusion and prevents development of coagulopathy.

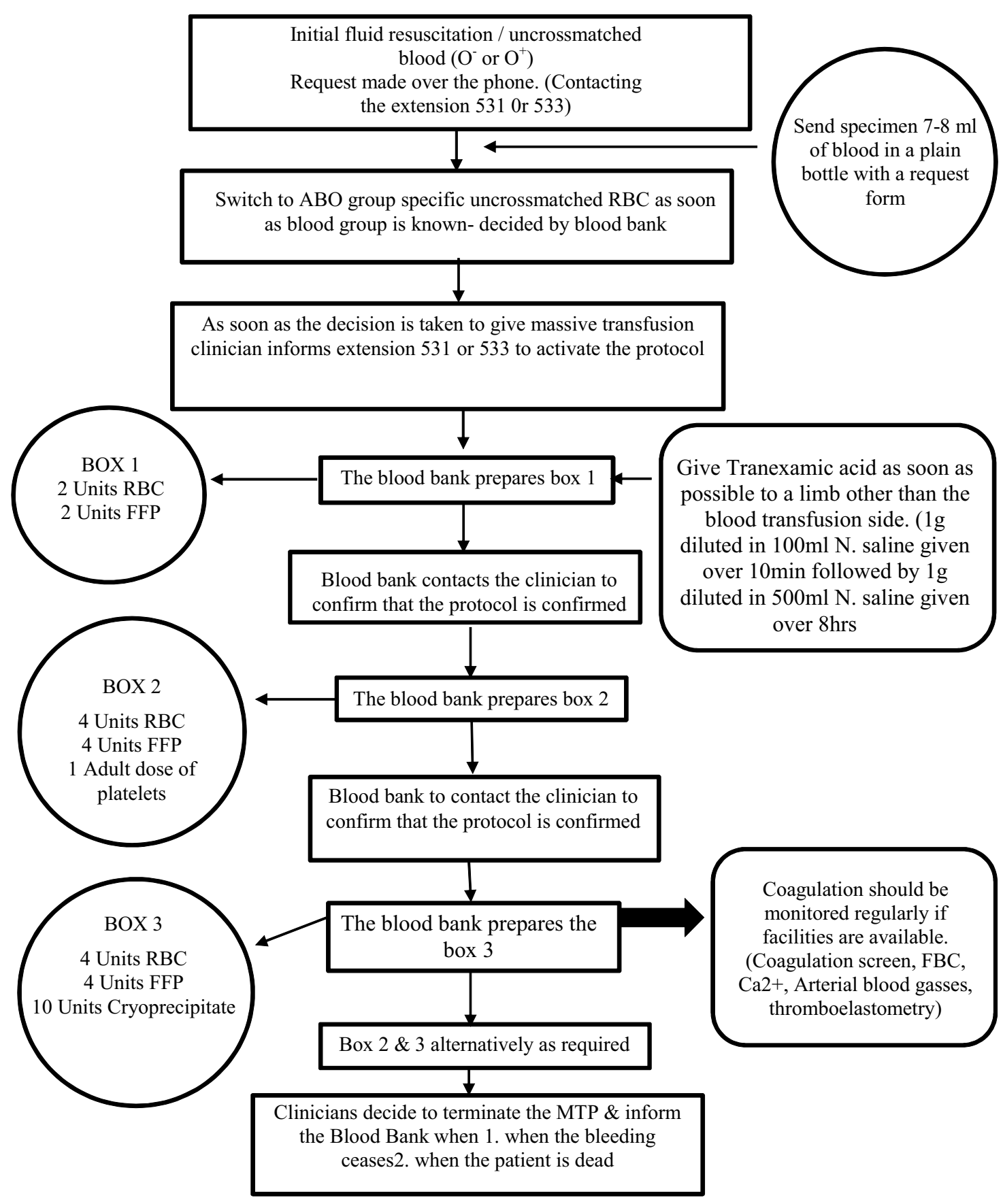

Figure 1. Flow Chart of Massive Transfusion Protocol-Colombo South Teaching Hospital

\section{Conclusion}

Severe bleeding in trauma carries high mortality. Apart

A ratio of 1:1:1, of packed red cells: FFP: apharesis platelet is being employed in our protocol. 
Implementation of this protocol requires close collaboration of surgeons, anaesthetists, transfusion specialists and haematologists. As the number of patients who require massive transfusion in a single centre is small, we need several years of implementation in several major centres in Sri Lanka to see the benefit and difficulties in implementing it. By introducing MTP, we hope to disseminate the knowledge about this and encourage others to use it.

With maturity of the system, we expect improvement in methodology of transfusions reducing the overall mortality in Sri Lanka.

\section{References}

1. Malone DL, Hess JR, Fingerhut A. Massive transfusion practices around the globe and a suggestion for a common massive transfusion protocol. Journal of Trauma-Injury Infection \& Critical Care 2006; 60: S 91-S 96

2. Hewitt PE, Machin SJ. ABC Transfusion, Massive blood transfusion. ABC of Transfusion, London: BMJ Publishing Group. 1990;300:107-109

3. Juan CD, John PH, Georgia W, et al. Review of current blood transfusions strategies in a mature Level I trauma center: Were we wrong for the last 60 years? Journal of Trauma-Injury Infection \& Critical Care 2008;65:272278

4. Holcomb JB, Wade CE, Michalcek JE, et al. Increased plasma and platelets to red blood cells ratio improves outcome Ann: Surgery. 2008;248:447-458

5. Borgman M A, Matthew A, Spinella, et al. The ratio of blood products transfused affects mortality in patients receiving massive transfusions at a combat support hospital. Journal of Trauma-Injury Infection \& Critical Care 2007;63 (4):805-813
6. Hoyt DB, Bulger EM, Knudson MM, et al. Death in the operating room; an analysis of center experience. Journal of Trauma-Injury Infection \& Critical Care 1994; 37 (3):426-432.

7. Karim Brohi, Jasmin Singh, Mischa Heron, et al. Acute traumatic coagulopathy. Journal of Trauma-Injury Infection \& Critical Care 2003;54(6)

8. Juan CD. Damage control resuscitation: addressing trauma-induced coagulopathy. British Journal of Hospital Medicine Jan 2009;70 (1):23-25

9. $\mathrm{Ng}$ KFJ, Lom CCK, Chan LC. In vivo effect of haemodilution with saline on coagulation. British Journal of Anesthesia 2002;88:475-480

10. Cotton BA, Jeffrey S, Guy JAM,et al. Cellular metabolic and systemic consequences of aggressive fluid resuscitation strategies; SHOCK 2006;26:115-121

11. Bicknell WH,Matthew J, Pepe PE. Immediate versus delayed fluid resuscitation for hypotensive patients with penetrating torso injuries. New England Journal of Medicine 1994;331:1105-1109

12. Bryan A. Cotton, Brigham K. Au, Thimothy C. Nunez. Predefined massive transfusion protocols are associated with a reduction in organ failure and postinjury complications. Journal of Trauma-Injury Infection \& Critical Care 2009;66:41-49

13. Dente CJ, Shaz BH,Nicholas J M, et al. Improvements in early mortality and coagulopathy are sustained better in patients with blunt trauma after institution of a massive transfusion protocol in a civilian Level I trauma centre. Journal of Trauma-Injury Infection \& Critical Care 2009: 1616-1624

14. Nunez TC, Voskresensky IV, Dossett LA, Shinall R, Dutton WD, Cotton BA. Early prediction of massive transfusion in trauma: simple as ABC (assessment of blood consumption)? J Trauma 2009 Feb;66(2):346-52

15. The CRASH-2 collaborators. Effects of Tranexamic acid on death, vascular occlusive events, and blood transfusion in trauma patients with significant haemorrhage (CRASH-2): a randomised, placebocontrolled trial, Lancet 2010; 376: 23-32. 